\title{
Motor performance of children and adolescents with cerebral palsy during the execution of computer tasks with different peripherals
}

Marcelo Grandini Spiller ${ }^{1}$
https://orcid.org/0000-0001-9431-4016

Mauro Audi ${ }^{1}$

https://orcid.org/0000-0003-4056-9253

Lígia Maria Presumido Braccialli'

https://orcid.org/0000-0002-2540-3725
Universidade Estadual Paulista Júlio de Mesquita Filho, Faculdade de Filosofia e Ciências, Programa de Pós-graduação em Educação, Marília, São Paulo, Brasil.

Research support source: Conselho Nacional de Desenvolvimento Cientifico e Tecnológico - CNPq, Process $474184 / 2009-9$

Conflict of interests: Nonexistent

\section{(C) $\underset{\mathrm{BY}}{\mathrm{i}}$}

Received on: Februry 11, 2019

Accepted on: July 9, 2019

Corresponding address:

Lígia Maria Presumido Braccialli Avenida Hygino Muzzi Filho, 737

Bairro: Mirante

CEP 17.525-900 - Marília, São Paulo,

Brasil

E-mail: bracci@marilia.unesp.br

\section{ABSTRACT}

Objective: to assess the effectiveness of using different input devices for computer access by children and adolescents with cerebral palsy and to verify the relationship of performance with age and level of motor classification.

Methods: the study included 14 children and adolescents with cerebral palsy, aged 6 to 14 years, with Gross Motor Function Classification System (GMFCS) and Manual Ability Classification System (MACS) levels from I to V. The participants' performance was observed during the use of touch screen, conventional mouse and eye tracking mouse in performing specific tasks with softwares Discrete Aiming Task 2.0, Tracking Task 2.0 and Single Switch Performance Test (SSPT) 1.0. The following variables were analyzed: response time, frequency of errors, mean time to activate input devices, relationship between satisfaction with input devices and motor skill level.

Results: results indicated significant differences in response time, frequency of errors, and triggering mean time depending on the input device used, and correlation between the motor ability level and satisfaction, using the device, and between the level of manual ability and satisfaction with the mouse.

Conclusion: the eye tracking mouse and the touch screen were the most effective devices during the execution of tasks on the computer by study participants.

Keywords: Cerebral Palsy; Self-Help Devices; Special Education; Information Technology 


\section{INTRODUCTION}

Computers have been highlighted as important assistive technology devices (AT) as they enable enhanced participation of children with severe motor and communication impairments at school, in daily leisure and social life activities ${ }^{1-3}$.

Paradoxically, computers are under-utilized by these individuals, despite a wide range of available AT devices ${ }^{1.4}$. In addition, school-aged children with cerebral palsy (CP) spend less time on computers than children with typical development ${ }^{4}$ due to difficulties in accessing computers: (a) lack of knowledge about available resources; (b) few trained professionals to use the devices; (c) lack of resource funding for this population; (d) complexity inherent to their condition, (e) inadequate assessments; (f) lack of follow-up'.

Although conventional interfaces for computer access, such as mouse, keyboards or joysticks are difficult to control ${ }^{5}$, people with motor impairment in the upper limbs still use the conventional, standard technology $\mathrm{y}^{6,7}$.

A variety of interaction methods and devices are currently available to improve physical access to computers; however, some barriers persist to using these technologies: insufficient funding, lack of staff training, negative attitudes in school, and high cost ${ }^{8}$. Moreover, it is necessary to develop computer interfaces that meet the needs of all individuals, that is, technology that accommodates a diversity of needs ${ }^{9}$.

Davies et al. ${ }^{6}$ interviewed young people with upper limb difficulties due to $\mathrm{CP}$, and identified that most of them prefer the conventional mouse to access computers rather than the hand for cursor movement and finger click for target selection. The study suggested that there are a number of reasons why young people with $\mathrm{CP}$ use the conventional or modified mouse, which include the lack of assistive technology provided in an education setting and fewer restrictions at different access points. Therefore, the more pragmatic decision is to use a conventional mouse in all locations.

Controlling the mouse requires users to have precise movements. Even people without disabilities cannot use the mouse perfectly without errors or additional tests at their first time. To access files on the computer with the conventional mouse, one must perform the tasks of point and click. Performance on these tasks is hampered by many factors, including fatigue, precision, position and hand-eye coordination ${ }^{8}$.

By using the conventional interface, a disabled person often needs to adopt inadequate posture patterns that increase their physical wear and tear. Thus, these users point out that although the computer increases their independence and control of activities, the effort to use this technology is sometimes greater than the benefits it brings?

On the other hand, developing software to improve the accessibility of input devices for disabled users in the upper limbs has been a concern. Recently, touch screen, switchers by body interaction and voice access devices have been incorporated into cell phones and other devices based on technology such as Microsoft Kinect ${ }^{4}$. Kinect is a motion sensor that allows access to games, Xbox consoles, without the use of controls or joysticks.

A study of a rehabilitation program of computer access with the use of a switch with six children with cerebral palsy who were unable to use the traditional mouse found it to be adequate and accessible. All children increased participation in the intervention phase and generalized to their home activities ${ }^{10}$.

Access to the computer through touchscreen technology has increased and improved in relation to sensitivity and availability over the past decade. However, for individuals with severe physical disabilities, these devices seem to remain largely inaccessible. Users with disabilities have reported usage limitations, especially regarding alternating controls and stored gestures; the adjustment of the click time, the inability to toggle or adjust the sliding functionality, the impossibility of modifying the location, size, shape and orientation of many buttons and toolbars ${ }^{11}$.

The latest computer access devices are based on webcams or infrared sensors. This type of interface seems to be a more natural way of pointing, in the same way as people tend to look at the object they want to interact with and do not require any bodily contact from the user. In these approaches, the system can control either the head movement, or the movement of the pupil in relation to the head. Other approaches do not use video images but detect user intentions by measuring cornea-retinal activity or by biological signals such as electroencephalographic activity or muscle activity 5 .

Many questions have been discussed regarding eye tracking and head tracking devices. The clinical training and the ongoing support required for this technology to support communication needs over time are yet to be understood, information is insufficient on how adverse environmental conditions affect the stability of the technology and the performance of the physical requirements of the long-term use of these systems, and 
how much time per day these individuals can spend using this technology to support their communication needs and computer access ${ }^{12}$.

In a study carried out with two students with cerebral palsy of athetoid type concluded that the camera mouse, computer access device through the head or face movements, was advantageous in relation to the use of a computer access device through eye control. As for the device with the eye control, due to involuntary movements, the participants continuously moved out of the capture field, which hindered performance ${ }^{13}$.

Another study carried out with seven students with cerebral palsy, aged between 5 and 15 years, who needed full assistance in all activities at school indicated that the use of a computer control device by gaze associated with a communication software may favor inclusion, and the students were able to control the computer and express themselves. However, the drawbacks pointed out by the teachers included the time to learn to control a computer by gaze and the individual adaptations to be made for each student ${ }^{14}$.

A study investigated the impact of computer control technology by gaze at the daily activities of children with severe motor and communication impairments. The research concluded that this type of technology had a positive impact on the repertoire and the opportunities for activities ${ }^{3}$.

To this date, the process of selecting a computer access device has been carried out by trial and error due to the lack of a valid predictive model to direct this process. In this context, the purpose of this study was to assess the effectiveness of using different input devices for computer access by children and adolescents with cerebral palsy, and to verify the relationship between performance with different devices and age and level of motor classification.

\section{METHODS}

A study with a quasi-experimental methodological design with a convenience sample. The project was received by the Research Ethics Committee of the Faculty of Philosophy and Sciences - UNESP Marília, SP, Brazil, and approved under number 0957/2014.

\section{Participants}

The study consisted of 14 individuals, children and adolescents diagnosed with cerebral palsy, 10 males and 4 females, aged 7 to 14 years (Figure 1). The inclusion criterion was: clinical diagnosis of cerebral palsy, without visual alterations that prevented performing the activities, understanding the commands to perform the tasks and being a computer user. All those responsible for the participants signed the Term of Informed Consent, and the Term of Informed Assent was read to all participants who agreed to participate. 


\begin{tabular}{|c|c|c|c|c|c|c|c|c|}
\hline Participant & Age & Sex & GMFCS & MACS & Topographic Diagnosis & Mouse & $\begin{array}{c}\text { Touch } \\
\text { Screen }\end{array}$ & $\begin{array}{c}\text { Eye } \\
\text { Tracking }\end{array}$ \\
\hline P1 & 6 & F & II & I & Spastic hemiplegic & C & C & C \\
\hline P2 & 14 & M & V & V & Choreo-athetoid quadriplegic & NC & C & C \\
\hline P3 & 10 & M & V & V & Spastic quadriplegic & NC & NC & C \\
\hline P4 & 8 & M & IV & II & Spastic Diplegic & C & C & C \\
\hline P5 & 10 & M & V & V & Athetoid quadriplegic & NC & C & NC \\
\hline P6 & 13 & F & V & V & Athetoid quadriplegic & NC & C & C \\
\hline P7 & 13 & M & I & I & Spastic hemiplegic & C & C & C \\
\hline P8 & 9 & M & IV & III & Spastic quadriplegic & NC & C & C \\
\hline P9 & 13 & M & I & I & Spastic Diplegic & C & C & C \\
\hline P10 & 10 & M & III & IV & Spastic Diplegic & C & C & C \\
\hline P11 & 10 & F & II & III & Spastic Diplegic & C & C & C \\
\hline P12 & 9 & M & I & II & Spastic hemiplegic & C & C & C \\
\hline P13 & 14 & M & III & II & Spastic Diplegic & C & C & C \\
\hline P14 & 6 & F & II & I & Spastic hemiplegic & C & C & C \\
\hline
\end{tabular}

${ }^{*} \mathrm{C}=$ can use peripheral; $\mathrm{NC}=$ can not use peripheral

GMFCS $=$ Gross Motor Function Classification System; MACS = Manual Ability Classification Systeml

Figure 1. Study participants' characteristics in relation to cerebral palsy classification, topographic diagnosis and the ability to use peripherals for computer access

\section{Procedures for data collection}

For data collection, each participant was positioned in a chair with adequate posture that favored gazing the monitor of a touch screen notebook, which was placed on a stand on the table in front of them. The height of the table, the distance of the monitor from the participant's head, and the angle of the notebook stand were measured to ensure that all interventions occurred in the same position. Regarding the positioning, each participant was accommodated in an adapted chair so that the hip, knees and ankles were at $90^{\circ}$, and a table with semicircle cutout was placed in front of them, the table height was adjusted so that each participant did not make any excessive flexion of shoulders to support their upper limb.

\section{Experimental Protocol}

The dominant upper limb was positioned at a demarcated starting point on the table and the other limb rested on the table so as not to interfere with the movement of the dominant limb while performing the activity with the mouse and screen touching. The task was to activate the target on the computer screen as quickly as possible, in each specific task with each software.
The computer monitor was positioned so that the center of the screen was placed at a distance corresponding to the maximum range of each participant. Peak range is an anthropometric measurement that consists of determining a hypothetical axis of the upper limb centered at the shoulder joint and from this point a ray, equal to the length of the upper limb, is drawn 15,16 to ensure that all the participants had access to the target when they used the touch screen.

For this study, three input devices were used: mouse, touch screen and Tobii PCEye GO eye tracking mouse, which were used during activities in the software Discrete Aiming Task v.2.0, Tracking Task v.2.0 and Single Switch Performance Test (SSPT).

The eye tracking mouse was calibrated for each participant, before starting data collection. Then, each child practiced, for at least three days, the activities proposed in the software Discrete Aiming Task v.2.0, Tracking Task v.2.0 and Single Switch Performance Test (SSPT), with the three types of input devices, mouse, eye tracking mouse Tobii PCEye GO and touch screen.

In the Discrete Aiming Task software, the participant performed the task of clicking inside two thin plates, alternately. The total number of two clicks to perform the task was stipulated, and the total time to perform 
the task was computed. In the Tracking Task software, the participant had to keep the mouse cursor inside a blue circle that changed direction several times in the computer screen for 10 seconds. For the Single Switch Performance Test (SSPT) software, the click was performed every time a yellow screen appeared on the computer.

Data collection was performed in one single day, previously scheduled. During data collection, the participant performed all the software tasks, previously trained. The order of execution was at random, by raffle, before each collection.

\section{Data analysis procedures}

A descriptive statistical analysis was performed through mean and standard deviation for the studied variables. For the variables total response time (s), frequency of errors during the execution of the task and mean time of activation, similarity between the groups was verified through the Friedman test for dependent samples. When there was a statistically significant difference, a two-to-two comparison was made using the Dunn's Comparison test. To verify whether there was a relationship between the variables, severity level (GMFCS and MACS) and age, Spearman's Correlation test was used with the input devices. It was considered: $r=0.10$ to 0.30 (weak); $r=0.40$ to 0.6 (moderate); $r$ $=0.70$ to 1 (strong). Data were checked for normality using the Kolmogorov-Smirnov's test. For all tests, the significance level of $5 \%$ probability for rejection of the null hypothesis was adopted.

\section{RESULTS}

Results were presented in relation to the variables: 1) total response time; 2) frequency of errors; 3 ) mean time for activating the input devices; 4) relationship between satisfaction with the input devices and level of motor ability.

The total response time during the use of the Discrete Aiming Task v.2.0 software $(p=0.0479) ;(p=$ $0.0022)$ during the execution of SSPT activity and the frequency of errors during the use of the Tracking Task v.2.0 software ( $p=0.0092)$ showed significant differences (Table 1).

Table 1. Results obtained for the variables during computer activation with the different input devices, using the Friedman test

\begin{tabular}{lccc}
\hline & $\begin{array}{c}\text { total response time }(\mathbf{s}) \\
\text { mean }(\mathbf{S D}) *\end{array}$ & $\begin{array}{c}\text { Tmean time of activation }(\mathbf{s}) \\
\text { mean }( \pm \mathbf{S D}) * *\end{array}$ & $\begin{array}{c}\text { frequency of errors } \\
\text { mean }( \pm \mathbf{S D}) * * *\end{array}$ \\
\hline Touch Screen & $2.89( \pm 2.02)$ & $36.14( \pm 37.16)$ & $7.83( \pm 5.02)$ \\
Mouse & $4.66( \pm 3.35)$ & $35.3( \pm 31.19)$ & $11.4( \pm 5.93)$ \\
Eye Tracking & $5.85( \pm 4.65)$ & $141.30( \pm 41.66)$ & $3.71( \pm 3.07)$ \\
\hline
\end{tabular}

${ }^{*} p=0.0479{ }^{* *} p=0.0022{ }^{* * *} p=0.0092$

$\mathrm{SD}=$ standard deviations

The comparison between the different devices indicated that there is a significant difference for: the variable total response time during the activation task executed in the Discrete Aiming Task v.2.0 when the touch and the eye tracking mouse were used; mean time of activation of the input devices during the execution of the activity with SSPT when comparing mouse and eye tracking mouse; and error frequency during the use of Tracking Task v.2.0 when comparing the mouse and eye tracking mouse and activation by touch and eye tracking mouse (Table 2). 
Table 2. Two-to-two comparison, using Dunn's test, for the variables during the tasks of activating the computer with the different devices

\begin{tabular}{|c|c|c|c|}
\hline & & Touch Screen & Eye Tracking \\
\hline \multirow{2}{*}{ total response time (s) } & mouse & $>0.05$ & $>0.05$ \\
\hline & touch screen & - & $<0.05^{\star}$ \\
\hline \multirow{2}{*}{ mean time of activation (s) } & mouse & $>0.05$ & $<0.01^{* *}$ \\
\hline & touch screen & - & $>0.05$ \\
\hline \multirow{2}{*}{ frequency of errors } & mouse & $>0.05$ & $<0.01 * *$ \\
\hline & touch screen & - & $<0.05^{\star}$ \\
\hline
\end{tabular}

significant $p$-value * very significant p-value **

Spermann's test indicated a negative correlation between the level of motor ability and satisfaction with touch and mouse and a negative correlation between the level of manual ability (MACS) and satisfaction with the mouse (Table 3).

Table 3. Correlation between variables age, Gross Motor Function Classification System, Manual Ability Classification System and satisfaction with the input devices, using Spermann's test

\begin{tabular}{lccc}
\hline & Eye Tracking & Touch Screen & Mouse \\
& $\mathbf{p}(\mathbf{r})$ & $\mathbf{p}(\mathbf{r})$ & $\mathbf{p}(\mathbf{r})$ \\
\hline Age & $0.138(0.40)$ & $0.786(-0.07)$ & $0.59(-0.14)$ \\
GMFCS & $0.807(0.068)$ & $0.03(-0.55)^{*}$ & $0.01(-0.60)^{*}$ \\
MACS & $0.267(0.30)$ & $0.1936(-0.41)$ & $0.01(-0.63)^{*}$ \\
\hline
\end{tabular}

significant $p$-value *

GMFCS $=$ Gross Motor Function Classification System; MACS = Manual Ability Classification System

\section{DISCUSSION}

Results indicated that the eye tracking mouse has a positive effect on the performance of children with cerebral palsy, as the frequency of error was lower with the use of this device, on the other hand, the time to execute the task was longer. These results are similar to those found by other authors, who indicated that this technology contributes to independence in the execution of tasks, but the disabled user, as well as their teachers, caregivers and therapists need training ${ }^{3,14}$.

The success of using eye tracking mouse when compared to the conventional mouse in target selection tasks in individuals with motor impairments can also be seen in the study by Mollenbach, Stefansson and Hanson. The authors concluded that for the tasks of navigating and searching the responses were indistinguishable, however, in target selection tasks, the eye tracking mouse was $16 \%$ faster than the traditional mouse ${ }^{17}$.
Satisfaction results also demonstrated that most participants seem to be satisfied with the touch screen and the eye tracking mouse, which corroborates the findings of other researchers ${ }^{18}$.

The results found in this study differ from the findings by Davies et al. who reported that most young people with CP still prefer the use of the conventional mouse commonly used to access the computer and the touch screen for targeting ${ }^{6}$. Although the eye tracker is not routinely available in the school, home, or therapeutic settings, the results indicated that this population could benefit from such devices.

Despite the greater efficiency and satisfaction with the use of the eye tracking mouse, when the frequency of error is referenced, users tended to perform activities more quickly when using the conventional mouse. This information seems to be related to the availability and training of conventional mouse use in different settings, although they are ineffective for this population. 


\section{CONCLUSIONS}

Regarding the frequency of error during the execution of a computer task, children with cerebral palsy performed better with the use of input devices with the eye tracking mouse and the touch screen. However, in relation to the task execution time, the participants were more efficient using the conventional mouse. The results also indicated a negative correlation between participants' satisfaction and the use of the conventional mouse.

\section{ACKNOWLEDGEMENTS}

CNPq (Scientific and Technological Development Nacional Council) for the granted financial assistance.

\section{REFERENCES}

1. Hoppestad BS. Inadequacies in computer access using assistive technology devices in profoundly disabled individuals: an overview of the current literature. Disabil Rehabil Assist Technol. 2007;2(4):189-99.

2. Hung Y-C, Charles J, Gordon AM. Bimanual coordination during a goal-directed task in children with hemiplegic cerebral palsy. Dev Med Child Neurol. [Internet]. novembro de 2004 [cited 2010 sept 30];46(11):746-53. Available from: http://www. ncbi.nlm.nih.gov/pubmed/19851759

3. Borgestig M, Sandqvist J, Ahlsten G, Falkmer T, Hemmingsson H. Gaze-based assistive technology in daily activities in children with severe physical impairments-An intervention study. Dev Neurorehabil [Internet]. 2016 [cited 2017 jan 10];8423(April):1-13. Available from: http://www. tandfonline.com/doi/full/10.3109/17518423.2015.11 32281

4. Chou C-J, Huang W-N, Tsai M-H. Browsing without clicking - two proposals of web interface design for universal accessibility. Procedia Manuf Elsevier BV [Internet]; 2015 [cited 2016 Aug 16]; 3(Ahfe):6290-7. Available from: http://www.sciencedirect.com/ science/article/pii/S2351978915008100

5. Raya R, Roa JO, Rocon E, Ceres R, Pons JL. Wearable inertial mouse for children with physical and cognitive impairments. Sensors Actuators, A Phys Elsevier BV [Internet]; 2010 [cited $2010 \mathrm{dec}$ 15];162(2):248-59. Available from: http://dx.doi. org/10.1016/j.sna.2010.04.019

6. Davies TC, Mudge S, Ameratunga S, Stott NS. Enabling self-directed computer use for individuals with cerebral palsy: a systematic review of assistive devices and technologies. Dev Med Child Neurol [Internet]. 2010 [cited 2015 Aug 12];52(6):510-6. Available from: http://doi.wiley. com/10.1111/j.1469-8749.2009.03564.x

7. Dorrington P. User-centered design method for the design of assistive switch devices to improve user experience, accessibility and independence. $J$ usability Stud. 2016;11(2):66-82.

8. Almanji A, Davies C, Amor R. Examining dynamic control-display gain adjustments to assist mousebased pointing for youths with cerebral palsy. Int $\mathrm{J}$ Virtual Worlds Hum Comput Interact [Internet]. 2015 [cited 2016 aug 16];3(1). Available from: http://vwhci.avestia.com/2015/001.html

9. Stephanidis C. User interfaces for all - new perspectives into human-computer interaction. In: User Interfaces for all - Concepts, Methods, and Tools. 2001. p. 3-17.

10. Stasolla F, Damiani R, Perilli V, D'Amico F, Caffo $A O$, Stella $A$ et al. Computer and microswitchbased programs to improve academic activities by six children with cerebral palsy. Res Dev Disabil. 2015;45-46:1-13.

11. Wiegand K, Patel R. Impact of motor impairment on full-screen touch interaction. J Technol Pers wirh Disabil. 2015;3(22):58-76.

12. Fager $S$, Beukelman DR, Fried-Oken $M$, Jakobs $T$, Baker J. Acess interface strategies. Assist Technol. 2011;24(1):25-34.

13. Man DWK, Wong M-SL. Evaluation of computeraccess solutions for students with quadriplegic athetoid cerebral palsy. Am J Occup Ther [Internet]. 2007 [cited 2016 Aug 16];61(3):355-64. Available from:: http://www.ncbi.nlm.nih.gov/ pubmed/17569393

14. Rytterström $P$, Borgestig $M$, Hemmingsson $H$. Teachers' experiences of using eye gaze-controlled computers for pupils with severe motor impairments and without speech. Eur J Spec Needs Educ [Internet]. 2016 [cited 2017 jan 17];31(4):506-19. Available from: https://www.tandfonline.com/doi/ full/10.1080/08856257.2016.1187878

15. Jarosz E. Determination of the workspace of wheelchair users. Int $\mathrm{J}$ Ind Ergon [Internet]. fevereiro de 1996 [cited 2017 jan 17];17(2):123-33. Available from: http://linkinghub.elsevier.com/ retrieve/pii/0169814195000445

16. Nowak E. The role of anthropometry in design of work and life environments of the disabled 
population. Int J Ind Ergon [Internet]. 1996 [cited 2017 jan 17];17(2):113-21. Available from: http://linkinghub.elsevier.com/retrieve/ pii/0169814195000437

17. Mollenbach E, Stefansson T, Hansen JP. All eyes on the monitor: gaze based interaction in zoomable, multi-scaled information-spaces. Proc 13th Int Conf Intell user interfaces [Internet]. 2008 [cited 2017 jan 17];42:373-6. Available from: http://portal.acm.org/ citation.cfm? $\mathrm{id}=1378833$

18. Caligari M, Godi M, Guglielmetti S, Franchignoni F, Nardone A. Eye tracking communication devices in amyotrophic lateral sclerosis: Impact on disability and quality of life. Amyotrophic Lateral Sclerosis and Frontotemporal Degeneration. 2013 [cited 2017 jan 17];14(7-8):546-52. Available from: https://www. researchgate.net/publication/247770106_Eye_ tracking_communication_devices_in_amyotrophic_ lateral_sclerosis_Impact_on_disability_and_quality_ of_life 